\title{
Introduction to the special issue on autonomous agents for agent-based modeling
}

\author{
Virginia Dignum $^{1}$. Nigel Gilbert ${ }^{2}$. \\ Michael P. Wellman ${ }^{3}$
}

Published online: 8 September 2016

(C) The Author(s) 2016

Agent-based modeling (ABM) is a general approach to understanding social systems through simulation of interacting agents. An agent-based model is by definition a model of a multi-agent system (MAS), yet historically the ABM and MAS research communities have proceeded on nearly independent tracks. This special issue bridges the MAS and ABM communities, by collecting research contributions that serve goals of both fields.

Combining the benefits of ABM and MAS perspectives poses many technical and conceptual challenges, as are illustrated in the papers composing this issue. Validation of ABM models is one such challenge. Zhang et al. [6] address this by proposing a data-driven ABM framework in which an individual behavior model is acquired by machine learning techniques, deployed in multi-agent systems and validated using a sequence of collective adoption decisions. They apply this framework to forecasting individual and aggregate residential rooftop solar adoption in San Diego county and demonstrate that the resulting agent-based model successfully forecasts solar adoption trends and provides a meaningful quantification of uncertainty about its predictions.

Singh et al. [4] address a key integration challenge of coupling event-based BDI systems to time-stepped $\mathrm{ABM}$ systems by presenting a framework that allows belief-desire-intention (BDI) cognitive agents to be embedded in an ABM system. Architecturally, this means that BDI is used to model the brains of agents whose bodies exist in an ABM system. The architecture is flexible in that the ABM can still have non-BDI agents in the simulation, and the BDI-side can have agents that do not have a physical counterpart (such as an organization).

\footnotetext{
$凶$ Virginia Dignum

M.V.Dignum@tudelft.nl

Nigel Gilbert

N.Gilbert@surrey.ac.uk

Michael P. Wellman

wellman@umich.edu

1 Delft University of Technology, Jaffalaan 5, Delft, Netherlands

2 University of Surrey, Guildford, UK

3 University of Michigan, Ann Arbor, MI, USA
} 
The potential of the integration of $\mathrm{ABM}$ and MAS approaches to handle modeling and analysis of complex (socio-technical) systems is illustrated by the following three contributions. In their paper, Chliaoutakis and Chalkiadakis [1] combine ABM and MAS techniques to study social relations in ancient societies in archeology, in a simulation using autonomous utility-based agents. The model allows exploration of the transition from a simple to a more complex society. Their results show an impressive social sustainability when socio-economic models based on self-organization are used. Nardin et al. [2] illustrate the potential of complex normative architectures and norm-aware agents to understand the dynamics of organized criminal groups and extortion racket systems. This model enables the analysis of both agents' behaviors and the mental normative representations behind them. Parikh et al. [3] take a synthetic information approach, where multiple data sets are combined to construct a synthesized representation of the population of the region with accurate demographics and infrastructure information. The resulting agents' behaviors are represented using the options framework achieving behavior models of increasing complexity.

This special issue is accompanied by a position paper by Wellman, "Putting the agent in agent-based modeling" [5], based on his presentation on the occasion of his 2014 ACM/SIGAI Autonomous Agents keynote which addresses the challenge of model sensitivity to specific choices of agent behavior by using well-proven MAS methods to enrich the quality of ABM models.

\section{References}

1. Chliaoutakis, A., \& Chalkiadakis, G. (2016). Agent-based modeling of ancient societies and their organization structure. Autonomous Agents and Multi-Agent Systems, this issue.

2. Nardin, L. G., Andrighetto, G., Conte, R., Szekely, A., Anzola, D., Elsenbroich, C., Lotzman, U., Neumann, M., Punzo, V., \& Troitzsch, K. G. (2016). Simulating protection rackets: A case study of the sicilian mafia. Autonomous Agents and Multi-Agent Systems, this issue.

3. Parikh, N., Hayatnagarkar, H. G., Beckman, R. J., Marathe M. V., \& Swarup, S. (2016). A comparison of multiple behavior models in a simulation of the aftermath of an improvised nuclear detonation. Autonomous Agents and Multi-Agent Systems, this issue.

4. Singh, D., Padgham, L., \& Logan, B. (2016). Integrating bdi agents with agent based simulation platforms. Autonomous Agents and Multi-Agent Systems, this issue.

5. Wellman, M. (2016). Putting the agent back in agent-based modelling. Autonomous Agents and Multi-Agent Systems, this issue.

6. Zhang, H., Vorobeychik, Y., Letchford, J., \& Lakkaraju, K. (2016). Data-driven agent-based modeling, with application to rooftop solar adoption. Autonomous Agents and Multi-Agent Systems, this issue. 